Review

\title{
Exosomes Mediate the Intercellular Communication after Myocardial Infarction
}

\author{
Ming-Jie Yuan ${ }^{1}$, Taneen Maghsoudi ${ }^{2}$, Tao Wang ${ }^{2}$ \\ 1. Department of Cardiology, Renmin Hospital of Wuhan University, Wuhan 430060, P.R. China. \\ 2. Cardiovascular Research Center, University of Virginia, USA. \\ $\triangle$ Corresponding author: Ming-Jie Yuan, Ph.D.; M.D. Department of Cardiology, Renmin Hospital of Wuhan University Wuhan 430060, PR. China. Jiefang Rd, \\ 238\#, Wuhan City, Hubei province, China. Tel. \& Fax: 86-27-88040334 E-mail: yuanmj8341@163.com; my6t@services.virginia.edu. \\ (C) Ivyspring International Publisher. Reproduction is permitted for personal, noncommercial use, provided that the article is in whole, unmodified, and properly cited. See \\ http://ivyspring.com/terms for terms and conditions.
}

Received: 2015.10.12; Accepted: 2015.12.10; Published: 2016.02.01

\begin{abstract}
The mechanisms of cardiac repair after myocardial infarction (MI) are complicated and not well-understood currently. It is known that exosomes are released from most cells, recognized as new candidates with important roles in intercellular and tissue-level communication. Cells can package proteins and RNA messages into exosome and secret to recipient cells, which regulate gene expression in recipient cells. The research on exosomes in cardiovascular disease is just emerging. It is well-known that exosomes from cardiomyocyte can transfect endothelial cells, stem cells, fibroblasts and smooth muscle cells to induce cellular changes. After myocardial infarction (MI), the exosomes play important roles in local and distant microcommunication. Nowadays, exosomal microRNAs transportation has been found to deliver signals to mediate cardiac repair after MI. However, the exosomes quality and quantities are variable under different pathological conditions. Therefore, we speculate that the monitoring of the quality and quantity of exosomes may serve as diagnosis and prognosis biomarkers of $\mathrm{MI}$, and the study of exosomes will provide insights for the new therapeutics to cardiac remodeling after Ml.
\end{abstract}

Key words: exosomes, cardiac remodeling; microRNA; myocardial infarction.

\section{Introduction}

Myocardial infarction (MI) continues to be the most common cause of death worldwide [1]. Although treatments in acute phase, such as coronary bypass surgery, balloon dilatation of coronary vessels can alleviate the initial cardiac damage in the acute phase of MI, there is still a need of novel therapies to improve tissue repair after the acute phase. Recently, exosomes released from mammalian cells have been reported as a new tool for cell-to-cell communication, and some exosomes can inhibit cell apoptosis and increase cell proliferation $[2,3]$. These effects are favorable for the tissue repair after MI, thus, we proposed that exosomes may serve as a new therapeutic target to regulate cardiac remodeling after MI.

\section{Secretion and molecular composition of exosomes}

Exosomes are a subgroup of extracellular vesi- cles generally ranging in size from 40 to $200 \mathrm{~nm}$, released through exocytosis $[4,5]$ from many cell types including stem cells, endothelial cells, fibroblasts cells and tumors cells. Moreover, exosomes are detectable in body fluids, such as blood, urine, saliva, and breast milk. Exosomes contain a set of specific and similar surface proteins like CD9, CD63, CD81 etc [6]. The exosomes release from cell is regulated mainly by certain RAB GTPase (Rab27a/b and Rab35) [7]. On the other hand, the inducible secretion and protein content of exosomes is regulated by pathological changes in the environment, including hypoxia, fever, and ethanol treatment $[8,9]$. Exosomes can mediate local and systemic cell-to cell communication through proteins, mRNAs, and miRNAs delivery, which induce physiological changes in recipient cells $[10,11]$. The mechanisms of exosomes-induced signal transfer are not completely clarified. Recent reports suggest 
that it may go through receptor-ligand mediated interactions or exosomal internization, which eventually lead to release of the cargo directly into the cytoplasm of the recipient cell [12].

\section{Exosomal miRNAs transfer in MI}

Reports have suggested that the myocardial tissue secretes exosomes, and which could be an important mechanism involved in heterocellular communication in the adult heart, especially exosomes emerging in the border zone of MI [13-15]. In addition, it needs to be mentioned that the type and quantity of exosomes derived from the border zone and normal myocardium were different. Kanada reported that exosomes from the same source did not deliver functional nucleic acids, suggesting the selectivity and importance of exosomal miRNA transfer [16]. In a human study, miR-1 and miR-133a are increased in serum of patients with acute coronary syndrome. Furthermore, using a mouse MI model, the authors have shown that miR-133a is released in exosomes from cardiamyocytes under ischemia [17]. Experimental data further provided evidence that cardiac function were improved when given cardiac progenitor cells (CPCs) and hypoxia-inducible factor 1(HIF1). Exosomes secreted from HIF1 overexpressed endothelial cells (EC) can be taken up by the transplanted CPCs in the infarcted area and increase the levels of miR-126 and miR-210 in CPCs. This study sheds light on how miRNA transport via exosomes and can act as potential mechanism for molecular cross-talk [18]. MiR-214 has been shown to be upregulated in the heart after ischemia [19], it has also been shown to be secreted in exosomes from human endothelial cells [20]. Sahoo et al suggested that circulating human CD $34+$ stem cell-secreted exosomes are enriched with miR-126, and the level of miR-126 in the circulation can imply the mobilization and release of exosomes from stem cells [21]. Interestingly, a more recent study evaluated the exosomes secreted from CPCs; they found that 7 miRNAs were differentially expressed in exosomes secreted from CPCs under hypoxia and normoxia. The miR-103 and miR-105b were elevated in ECs and fibroblasts because these cells uptake the exosomes from CPCs [22]. All the above results suggest that exosomes transport miRNA information, and different cell-derived exosomes induced selective miRNA profile expression, which may protect cell from death in the ischemia

\section{Exosomes mediated cell-cell commu- nication}

Intercellular communication is essential for multicellular organisms to maintain vital function. The release of exosomes is an important style of contact between cells, with interaction and uptake by another cell. Exosomes have been shown to mediate communication between endothelial cells, smooth muscle cells, cardiac myocytes, stem cells and fibroblast [23-25]. It is demonstrated that the exosomes from cultured HL-1 murine cardiomyocytes contained 1595 different RNAs and 423 could be directly connected to a biological network, 33 genes coded for proteins. Moreover, these exosomes were internalized by fibroblasts when co-cultured, exosomal DNA transfer was possible into target fibroblasts, where exosomes stained for DNA were seen in the fibroblast cytosol and even in the nuclei [26]. These suggest a new concept in cardiomyocyte communication, proposing that exosomes are able to transfer protein or genetic information to recipient cells. Recently, Bang et al reported that miR-21* is selectively packaged into fibroblast-secrected exosomes, which can be taken up by cardiomyocytes and subsequently induce cardiac hypertrophy [23]. In another report, a $16-\mathrm{kDa}$ N-terminal prolactin fragment (16K PRL) not only induced the miR-146a expression in ECs, but also enhanced the release of miR-146a enriched exosomes from ECs, and the endothelial exosomes can be taken up by cardiomyocytes, furthermore, the miR-146a functions in cardiomyocyte by suppressing the expression of several target genes. Theses finding showed the communication between ECs and cardiomyocytes via exosomes[27]. In a rat diabetic model, miR-320 enriched exosomes secreted from cardiomyocytes could be taken up by ECs, and subsequently reduce tube formation. Recently, embryonic stem cell derived exosomes have been reported to augment CPCs survival, proliferation and cell cycle progression 8 weeks after MI in mouse models [28]. It is known that the cardiac remodeling after MI is complicated and the signal pathway interact with each other. The miRNAs could regulate the expression of target proteins and mediate cardiac protection after MI, but the key molecular is not clear. It is critical to identify the critical cell or blood-derived exosomes in the future study.

\section{Protective effects of exosomes in the MI}

Recent studies indicate that the beneficial effects of stem cells in the repair of injured myocardium are through the release of exosomes. A single intravenous bolus of exosomes derived from mesenchymal stem cells reduced infarct size by $45 \%$ in mice ischemia-reperfusion model. Moreover, exosomes treatment could reduce systemic inflammation in mice after myocardial ischemic/reperfusion [29]. It is interesting that CPCs derived exosomes can replicate CPC-induced therapeutic effect, and blockade of exosome secretion alleviated the protective effects of 
CPCs on the infarcted mouse heart, particularly, miR-146a has been shown to induce the major cardio-protective effects of CPCs secreted exosomes [30]. CPCs have also been shown to promote angiogenesis and cardiac myocyte survival in vitro by transferring exosomes [22, 31]. Further, Khan et al demonstrated that mouse ESC-derived exosomes possess ability to augment function in infarcted hearts as reflected by enhanced neovascularization, cardiomyocyte survival, and reduced fibrosis after MI [28]. In an ische$\mathrm{mia} /$ reperfusion rat model, exosomes from the blood healthy controls blood were reported to protect the ischemic myocardium by systemic injection, they showed that exosomes deliver endogenous protective signals to the myocardium by a pathway involving toll-like receptor 4 and classic cardio-protective heat shock protein 70 [32]. Exosomes secreted from GATA-4 overexpressing mesenchymal stem cells improved cardiac contractile function and reduced infarct size by releasing multiple miRs responsible for activation of the cell survival signaling pathway [33]. We can see that exosomes, especially secreted by CPC and BM-derived exosomes play protective roles in improving cardiac remodeling after MI.

\section{Conclusions and Perspective}

Exosomes may serve as the underlying factors by which damaged heart communicate with other cells, tissue and organs to initiate the repair process after MI. Exosomes derived from different conditions may contain different functional factors, which decide their properties. Because of the complex structure of exosomes, whether the special intervention of exosomes production during disease is able to have therapeutic effects is not clear. The transfer of miRNA is selective by exosomes[34], but we do not know how miRNAs are specifically packaged into exosomes and transfer to targeted cells. A recent paper published in Nature demonstrated that exosomes derived glypican-1 could be detects in early pancreatic cancer[35]. Targeting miRNA has been shown to be beneficial in a clinical trial [36]. Overall, exosomes secretion and uptake is tightly regulated, and understanding the underlying mechanisms will be important to control the cardiac injury repair. Conclusively, monitoring the quality and quantity of exosomes after MI may serve as new factor for disease evaluation and prognosis prediction, and the study of exosomes may identify novel clinical biomarkers and provide insights for new therapeutics to cardiac remodeling after MI.

\section{Acknowledgments}

This study was partially supported by National Natural Science Foundation of China (No. 81300315)

\section{Competing Interests}

The authors have declared that no competing interest exists.

\section{References}

1. Mozaffarian D, Benjamin EJ, Go AS, Arnett DK, Blaha MJ, Cushman M, et al. Heart Disease and Stroke Statistics-2015 Update A Report From the American Heart Association. Circulation. 2015;131:E29-E322.

2. Ailawadi S, Wang $X, G u$ H, Fan GC. Pathologic function and therapeutic potential of exosomes in cardiovascular disease. Biochim Biophys Acta. 2015;1852:1-11.

3. Das S, Halushka MK. Extracellular vesicle microRNA transfer in cardiovascular disease. Cardiovasc Pathol. 2015;24:199-206.

4. Frydrychowicz M, Kolecka-Bednarczyk A, Madejczyk M, Yasar S, Dworacki G. Exosomes - structure, biogenesis and biological role in non-small-cell lung cancer. Scand J Immunol. 2015;81:2-10.

5. Sluijter JP, Verhage V, Deddens JC, van den Akker F, Doevendans PA. Microvesicles and exosomes for intracardiac communication. Cardiovasc Res. 2014;102:302-11.

6. Vlassov AV, Magdaleno S, Setterquist R, Conrad R. Exosomes: current knowledge of their composition, biological functions, and diagnostic and therapeutic potentials. Biochim Biophys Acta. 2012;1820:940-8.

7. Ostrowski M, Carmo NB, Krumeich S, Fanget I, Raposo G, Savina A, et al. Rab27a and Rab27b control different steps of the exosome secretion pathway. Nat Cell Biol. 2010;12:19-30; sup pp 1-13.

8. de Jong OG, Verhaar MC, Chen Y, Vader P, Gremmels H, Posthuma G, et al Cellular stress conditions are reflected in the protein and RNA content of endothelial cell-derived exosomes. J Extracell Vesicles. 2012;1.

9. [9] Malik ZA, Kott KS, Poe AJ, Kuo T, Chen L, Ferrara KW, et al. Cardiac myocyte exosomes: stability, HSP60, and proteomics. Am J Physiol Heart Circ Physiol. 2013;304:H954-65.

10. Valadi H, Ekstrom K, Bossios A, Sjostrand M, Lee JJ, Lotvall JO. Exosome-mediated transfer of mRNAs and microRNAs is a novel mechanism of genetic exchange between cells. Nat Cell Biol. 2007;9:654-9.

11. Peinado H, Aleckovic M, Lavotshkin S, Matei I, Costa-Silva B, Moreno-Bueno $\mathrm{G}$, et al. Melanoma exosomes educate bone marrow progenitor cells toward a pro-metastatic phenotype through MET. Nat Med. 2012;18:883-91.

12. O'Loughlin AJ, Woffindale CA, Wood MJ. Exosomes and the emerging field of exosome-based gene therapy. Curr Gene Ther. 2012;12:262-74.

13. Barile L, Gherghiceanu M, Popescu LM, Moccetti T, Vassalli G. Ultrastructural evidence of exosome secretion by progenitor cells in adult mouse myocardium and adult human cardiospheres. J Biomed Biotechnol. 2012;2012:354605.

14. Manole CG, Cismasiu V, Gherghiceanu M, Popescu LM. Experimental acute myocardial infarction: telocytes involvement in neo-angiogenesis. J Cell Mol Med. 2011;15:2284-96.

15. Emanueli C, Shearn AI Angelini GD, Sahoo S. Exosomes and exosomal miRNAs in cardiovascular protection and repair. Vascul Pharmacol. 2015;71:24-30.

16. Kanada M, Bachmann MH, Hardy JW, Frimannson DO, Bronsart L, Wang A, et al. Differential fates of biomolecules delivered to target cells via extracellular vesicles. Proceedings of the National Academy of Sciences of the United States of America. 2015;112:E1433-E42.

17. Kuwabara $Y$, Ono $K$, Horie $T$, Nishi $H$, Nagao $K$, Kinoshita $M$, et al. Increased microRNA-1 and microRNA-133a levels in serum of patients with cardiovascular disease indicate myocardial damage. Circ Cardiovasc Genet. 2011;4:446-54.

18. Ong SG, Lee WH, Huang M, Dey D, Kodo K, Sanchez-Freire V, et al. Cross talk of combined gene and cell therapy in ischemic heart disease: role of exosomal microRNA transfer. Circulation. 2014;130:S60-9.

19. Aurora AB, Mahmoud AI, Luo X, Johnson BA, van Rooij E, Matsuzaki S, et al. MicroRNA-214 protects the mouse heart from ischemic injury by controlling $\mathrm{Ca}(2)(+)$ overload and cell death. J Clin Invest. 2012;122:1222-32.

20. [20] van Balkom BW, de Jong OG, Smits M, Brummelman J, den Ouden K, de Bree PM, et al. Endothelial cells require miR-214 to secrete exosomes that suppress senescence and induce angiogenesis in human and mouse endothelial cells. Blood. 2013;121:3997-4006, S1-15.

21. Sahoo S, Klychko E, Thorne T, Misener S, Schultz KM, Millay M, et al. Exosomes from human $\mathrm{CD} 34(+)$ stem cells mediate their proangiogenic paracrine activity. Circ Res. 2011;109:724-8.

22. Gray WD, French KM, Ghosh-Choudhary S, Maxwell JT, Brown ME, Platt $\mathrm{MO}$, et al. Identification of therapeutic covariant microRNA clusters in hypoxia-treated cardiac progenitor cell exosomes using systems biology. Circ Res. 2015;116:255-63.

23. Bang C, Batkai S, Dangwal S, Gupta SK, Foinquinos A, Holzmann A, et al Cardiac fibroblast-derived microRNA passenger strand-enriched exosomes mediate cardiomyocyte hypertrophy. J Clin Invest. 2014;124:2136-46.

24. Hergenreider E, Heydt S, Treguer K, Boettger T, Horrevoets AJ, Zeiher AM, et al. Atheroprotective communication between endothelial cells and smooth muscle cells through miRNAs. Nat Cell Biol. 2012;14:249-56. 
25. Wang X, Huang W, Liu G, Cai W, Millard RW, Wang Y, et al. Cardiomyocytes mediate anti-angiogenesis in type 2 diabetic rats through the exosomal transfer of miR-320 into endothelial cells. J Mol Cell Cardiol. 2014;74:139-50.

26. Waldenstrom A, Genneback N, Hellman U, Ronquist G. Cardiomyocyte microvesicles contain DNA/RNA and convey biological messages to target cells. PLoS One. 2012;7:e34653.

27. Halkein J, Tabruyn SP, Ricke-Hoch M, Haghikia A, Nguyen NQ, Scherr M, et al. MicroRNA-146a is a therapeutic target and biomarker for peripartum cardiomyopathy. J Clin Invest. 2013;123:2143-54.

28. Khan M, Nickoloff E, Abramova T, Johnson J, Verma SK, Krishnamurthy P, et al. Embryonic Stem Cell-Derived Exosomes Promote Endogenous Repair Mechanisms and Enhance Cardiac Function Following Myocardial Infarction. Circulation Research. 2015;117:52-64.

29. Arslan F, Lai RC, Smeets MB, Akeroyd L, Choo A, Aguor EN, et al Mesenchymal stem cell-derived exosomes increase ATP levels, decrease oxidative stress and activate PI3K/Akt pathway to enhance myocardial viability and prevent adverse remodeling after myocardial ischemia/reperfusion injury. Stem Cell Res. 2013;10:301-12.

30. Ibrahim AG, Cheng K, Marban E. Exosomes as critical agents of cardiac regeneration triggered by cell therapy. Stem Cell Reports. 2014;2:606-19.

31. Barile L, Lionetti V, Cervio E, Matteucci M, Gherghiceanu M, Popescu LM, et al. Extracellular vesicles from human cardiac progenitor cells inhibit cardiomyocyte apoptosis and improve cardiac function after myocardial infarction. Cardiovasc Res. 2014;103:530-41.

32. Vicencio JM, Yellon DM, Sivaraman V, Das D, Boi-Doku C, Arjun S, et al. Plasma Exosomes Protect the Myocardium From Ischemia-Reperfusion Injury. Journal of the American College of Cardiology. 2015;65:1525-36.

33. Yu B, Kim HW, Gong M, Wang JC, Millard RW, Wang YG, et al. Exosomes secreted from GATA-4 overexpressing mesenchymal stem cells serve as a reservoir of anti-apoptotic microRNAs for cardioprotection. International Journal of Cardiology. 2015;182:349-60.

34. Villarroya-Beltri C, Gutierrez-Vazquez C, Sanchez-Cabo F, Perez-Hernandez D, Vazquez J, Martin-Cofreces N, et al. Sumoylated hnRNPA2B1 controls the sorting of miRNAs into exosomes through binding to specific motifs. Nat Commun. 2013;4:2980.

35. Melo SA, Luecke LB, Kahlert C, Fernandez AF, Gammon ST, Kaye J, et al. Glypican-1 identifies cancer exosomes and detects early pancreatic cancer. Nature. 2015;523:177-U82.

36. Janssen HL, Reesink HW, Lawitz EJ, Zeuzem S, Rodriguez-Torres M, Patel K, et al. Treatment of HCV infection by targeting microRNA. N Engl J Med. 2013;368:1685-94. 